modification of standards for significant hazards. As well, social attitudes change and society generally becomes more risk-averse over time. An alternative approach is 'continuous quality improvement' (CQI), which is an on-going process for the optimisation of risk, efficiency of operations, and consumption of resources. The 'Deming Cycle' (Plan $\rightarrow \mathrm{Do} \rightarrow$ Study $\rightarrow$ Act $\rightarrow$ [repeat]), for example, is the standard management approach for quality assurance in the private sector. CQI has advantages over fixed standard setting in improving the quality of the environment and worker health and fits better with good management practices. The theoretical disadvantages may be business concern over an ever shifting target for compliance and the opportunity cost of making improvements when performance is already sufficient. However, in practice, CQI has shown such great benefit in improving the operations of enterprises from small business to large corporations that it is standard procedure and typically results in large unanticipated gains beyond quality, in efficient operations, lower cost, and reduced risk. This approach is rarely used in occupational health protection but it has been adopted as 'Best Available Control Technology' in other settings. In the United States, the mandated periodic review of ambient air quality standards by the EPA and of high-priority chemicals under the Lautenberg Chemical Safety Act are broadly compatible with CQI. The approach is also one means of effectively operationalizing the Precautionary Principle. It is suggested that CQI should be reconsidered as an alternative regulatory approach and adopted as a fundamental approach to risk management.

\section{WOMEN HEALTH AND WORK}

${ }^{1}$ Ravindra Kumar*, ${ }^{2}$ Pragati Patil, ${ }^{3}$ Shriniket Mishra, ${ }^{4}$ Anoop Singh. ${ }^{1}$ Hero MotoCorp Ltd, Neemrana, India; ${ }^{2}$ Indian Army, India; ${ }^{3}$ Chief Medical Officer, Hero MotoCorp Ltd; ${ }^{4}$ Assistant Professor, Dr SN Medical College Jodhpur

\subsection{6/oemed-2018-ICOHabstracts.443}

Introduction India, Women working on shop floor is new concept for Automobile industries. women taking up tools to partake in manufacturing of two-wheelers, hitherto a male bastion. As the call for women empowerment gets louder, Automobile two-wheeler manufacturers are responding with an aim to create a culture where women can stand shoulder to shoulder with their male counterparts to contribute to the growth of the company. Occupational health Centre is concerned about their health, a cross-sectional scientific study conducted in an automobile industry to assess Body Mass Index with an aim to make healthy work pattern suitable to their physiology.

Method Study was conducted on 27 randomly selected unmarried women work team member in age group of 19-24 years of frame assembly section in an automobile industry. Job was working on assembly line of two wheeler of automobile manufacturing industry. Parts pick from conveyor tray and to mounting on frame body of two-wheeler. Measure the weight and height and then calculate the BMI of the team member were computed at the time of joining and after 6 month of staring work, general clinical check-up including heart rate, and oxygen saturation and BP monitoring before and after the 6 month interval of work schedule.

Results Women working on shop floor mean age 21.5 years, mean weight $44.40 \mathrm{~kg}$ and mean height $161.66 \mathrm{~cm}$ and mean BMI 18.28 taking balance and with 8 hours shift duty, leave as per prorota basis, 48 hours work in one week. Review periodical medical examination after 6 month found mean height 161.66 and mean weight $50.30 \mathrm{~kg}$ and mean BMI 20.88 .

Conclusion This is first surveillance of women health status in automobile industry in India under occupational health physician. Taking balance diet with structured work pattern with appropriate work atmosphere result in improved average BMI.

\section{THE STRESS CHECK PROGRAM: AN EVALUATION OF THE FIRST-YEAR IMPLEMENTATION OF THE NEW NATIONAL WORKPLACE MENTAL HEALTH PROGRAM IN JAPAN}

${ }^{1} \mathrm{~N}$ Kawakami ${ }^{*},{ }^{1} \mathrm{~K}$ Imamura, ${ }^{1} \mathrm{Y}$ Asai, ${ }^{1} \mathrm{~K}$ Watanabe, ${ }^{2} \mathrm{~A}$ Tsutsumi, ${ }^{2} \mathrm{~A}$ Shimazu, ${ }^{2} \mathrm{~A}$ Inoue, ${ }^{3} \mathrm{H}$ Hiro, ${ }^{4} \mathrm{Y}$ Odagiri, ${ }^{5} \mathrm{~T}$ Yoshikawa, ${ }^{6} \mathrm{E}$ Yoshikawa. ${ }^{1}$ Graduate School of Medicine, The University of Tokyo, Tokyo, Japan; ${ }^{2}$ Kitasato University, Sagamihara, Japan; ${ }^{3}$ University of Occupational and Environmental Health, Kitakyushu, Japan; ${ }^{4}$ Tokyo Medical University, Tokyo, Japan; ${ }^{5}$ National Institute of Occupational Safety and Health, Kawasaki, Japan; ${ }^{6}$ Japanese Red Cross College of Nursing, Tokyo, Japan

\subsection{6/oemed-2018-ICOHabstracts.444}

Introduction The Stress Check Program mandates workplaces with 50 or more employees in Japan to provide their employees with an opportunity for a stress survey (the stress check), followed by a doctor interview for employees with high stress. Improvement of psychosocial work environment based on analysis of the stress check data is also recommended. The program went into its first year round between December 2015 and November 2016. The current paper overviews the implementation and effectiveness of the program in the first year.

Methods We reviewed reports from prefectural (local) labour bureaus wherever available. A prospective study of workplaces $(n=217)$ randomly selected was conducted to know the implementation and related costs. A prospective study of an internet sample of full-time workers $(n=2500)$ was also conducted to know the effects of the program for improving psychological distress. A workshop was held to listen to occupational health professionals and human resource personnel who engaged in running the program at their workplaces.

Result Prefectural labour bureaus reported that about $80 \%$ of workplaces implemented the program; the participation rate to the stress check was high (74\% to 94\%); only a small proportion of employees with high stress took the doctor interview. A similar pattern was found in the workplace cohort. In the cohort of workers, psychological distress was improved in a group that participated in the stress check and experienced work environment improvement significantly better than in a control group who did neither. Occupational health professionals had some difficulties in implementing the new program in the first year, while they planned to improve their practice in the second year.

Discussion The Stress Check Program was well implemented in the first year, while the beneficial effect of the program may be limited to those who experienced work environment improvement following the stress check.

\section{AN ERGONOMIST IN A CONTINUOUS IMPROVEMENT TEAM. LESSONS LEARNED FROM THE AVIATION MAINTENANCE INDUSTRY}

J Carmo*, M Lima. ,2UCS- Integrated Healthcare SA, TAP Portugal Group, Lisbon, Portugal

10.1136/oemed-2018-ICOHabstracts.445 